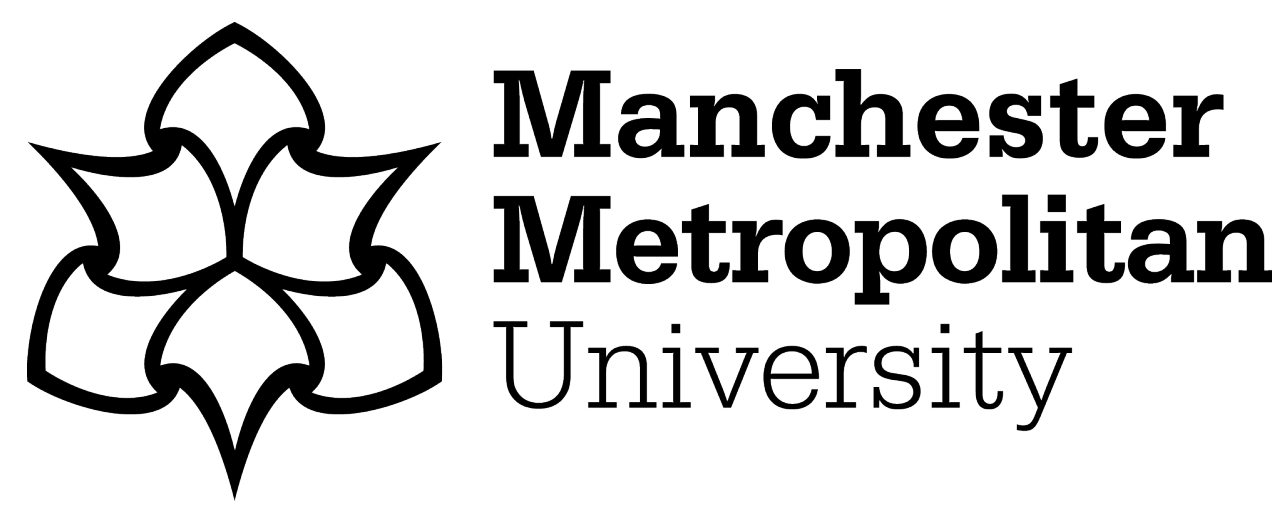

Khan, S, Alvi, AN, Javed, MA, Al-Otaibi, YD and Bashir, AK (2021) An efficient medium access control protocol for RF energy harvesting based loT devices. Computer Communications, 171. pp. 28-38. ISSN 0140-3664

Downloaded from: https://e-space.mmu.ac.uk/627610/

Version: Accepted Version

Publisher: Elsevier

DOI: https://doi.org/10.1016/j.comcom.2021.02.011

Usage rights: Creative Commons: Attribution-Noncommercial-No Derivative Works 4.0

Please cite the published version 


\title{
An efficient medium access control protocol for RF Energy Harvesting based IoT devices
}

\author{
Sangrez Khan ${ }^{\mathrm{a}}$, Ahmad Naseem Alvi ${ }^{\mathrm{a}}$, Muhammad Awais Javed ${ }^{\mathrm{a}, *}$, Yasser D. \\ Al-Otaibi ${ }^{\mathrm{b}, *}$, Ali Kashif Bashir ${ }^{\mathrm{c}}$ \\ ${ }^{a}$ Department of Electrical and Computer Engineering, COMSATS University Islamabad, \\ 45550, Islamabad \\ ${ }^{b}$ Department of Information Systems in Rabigh, King Abdulaziz University, Jeddah 21589, \\ Saudi Arabia \\ ${ }^{c}$ Department of Computing and Mathematics, Manchester Metropolitan University, United \\ Kingdom
}

\begin{abstract}
Energy efficiency is one of the major challenges in IEEE 802.15.4 based Internet of Things (IoT). In the Medium Access Control (MAC) layer of the IEEE 802.15.4 standard, Guaranteed Time Slot (GTS) are allocated to the IoT devices for data transmission. However, GTS allocation does not consider residual energy of IoT devices resulting in reduced life cycle of these devices. In this paper, we propose an efficient MAC protocol for RF harvesting based IoT devices. The proposed protocol uses residual energy based duty cycle adaptation to prioritize transmission of high energy devices, allowing low energy devices to harvest energy in the mean time. Simulation results show that the life cycle and transmitted data of IoT devices is improved up to $94 \%$ and $79 \%$ respectively by using the proposed protocol, as compared to the IEEE 802.15.4 standard.
\end{abstract}

Keywords: Internet of Things, Wireless Sensor Networks, Energy Harvesting, IEEE 802.15.4, GTS

${ }^{*}$ Corresponding authors: Muhammad Awais Javed and Yasser D. Al-Otaibi, email: awais.javed@comsats.edu.pk, yalotaibi@kau.edu.sa

Preprint submitted to Journal of ${ }^{A} T_{E} X$ Templates

April 30, 2021 


\section{Introduction}

The idea of robust and effective global computing infrastructure has been presented for many years. Wireless communication technology plays a vital role to enable such a widely interconnected computing environment [1, 2]. The 5 blending of sensing and wireless interaction has paved the path to the growth of the Internet of Things (IoT). One of the key components of the IoT network is wireless sensor nodes. These sensor nodes are battery operated with limited energy and low processing capabilities. These IoT based wireless sensor nodes send their information to their gateway either in single or in multi-hop fashion over unreliable links.

IoT technology has enabled a plethora of potential applications ranging from environment monitoring [3], tactical military application, Body Area Networks (BAN) [4, 5], home automation [6, vehicular ad hoc networks [7, 8, 9], smart cities 10, 11, and object tracking. As a result, it is expected that shortly, many IoT applications will be commercialized and available for public use.

Energy efficiency is a key challenge in IoT devices [12, 13]. Since the sensor devices are powered by the battery and once the energy of a node is exhausted, it will no more able to contribute to the network until the battery is changed or recharged. Replacing the exhausted batteries of sensors mass deployed in the outdoor situation is a difficult task and it also increases the operational expenditure. It is hence essential to conserve the battery power by improving the energy efficiency of the IoT sensor devices, resulting in increased lifetime [14, 15, 16. Different Medium Access Control (MAC) protocols have been proposed for IoT based WSN by emphasizing on their energy limitations. IEEE 802.15.4 standard was developed for such Wireless Personal Area Networks (WPAN) which are low power and require a low data rate with low processing. This is the reason, they are suited for WSNs and IoT applications [17.

It has been observed that some of the nodes in a network have to send and receive more data as compared to other nodes in that network. The node with an increased amount of transmitting and receiving data consumes its energy quickly 
as compared to the nodes with less amount of data transmitting or receiving. This causes an imbalance in the residual energies of nodes in a WPAN resulting in network instability. To increase the life cycle of all nodes, load balancing is required by allowing nodes to transmit or receive more data that have more remaining energy as compared to other nodes.

Recently, Energy Harvesting (EH) has been a vital research area to enhance the energy efficiency of IoT devices. EH refers to the mechanism of obtaining energy from the ambient surrounding like solar energy, kinetic energy (wind and mechanical vibration), and wireless energy (radio frequency). The energy from these sources can be transformed into electrical energy and can be directly consumed to operate the sensor node or first deposited in a storage battery and then supplied to the sensor nodes. Deployment of EH methods enables the sensor nodes to charge their on-board batteries in the working environment resulting in low operation cost and avoidance of network downtime. Consequently, energy harvesting based IoT devices has been gaining substantial attention.

It is to be noted that all energy harvesting techniques can not offer limitless energy for an unlimited period, at any particular time only a finite quantity of energy can be obtained [18. The energy level of sensor nodes increases when its energy consumption is less than its harvested energy. Generally, nodes increase their residual energy level when they are in sleep mode. However, their energy level decreases when they are transmitting or receiving any data as it consumes more energy during transmitting and receiving mode in comparison to harvested energy.

Energy-aware deep sleep mechanisms prefer the nodes with less residual energy to remain in sleep mode for energy conservation. In addition, it allows nodes to harvest energy during sleep mode. Nodes with low remaining battery power are better served if they are provided enough time to harvest energy and then proceed with their data transmission. This will save such nodes from battery depletion. The default IEEE 802.15.4 standard allows nodes to remain in sleep mode for a longer time even more than $99 \%$, that allows node to harvest more energy during sleep mode. Increase in sleep time decreases throughput 
with increased network delay and Quality of service (QoS) is compromised.

To meet these challenges, we propose an Efficient MAC protocol for IoT based sensor networks $\left(E-M A C_{I o T}\right)$, that helps PAN coordinator to adapt its duty cycle by considering the accumulated PAN energy without compromising the QoS. The proposed work based on RF based energy harvesting method that has been described in detail in prospect of IEEE 802.15.4 standard. In addition, it helps PAN coordinator to optimally scrutinizes GTS requesting nodes by considering their residual energy along with their data requests to increase their life cycle.

The main contribution of $\left(E-M A C_{I o T}\right)$ includes:

1. $\left(E-M A C_{I o T}\right)$ proposes an algorithm that allows the PAN coordinator to adjust its duty cycle based on the residual energy of the WPAN.

2. $\left(E-M A C_{I o T}\right)$ proposes another algorithm that helps the PAN coordinator to scrutinize GTS requesting nodes by considering their individual residual energy levels and their data requests.

3. $\left(E-M A C_{I o T}\right)$ is fully compatible with the IEEE 802.15.4 standard without compromising its existing parameters.

To evaluate the performance of $E-M A C_{I o T}$, a MATLAB based simulation environment is created and compared its performance with IEEE 802.15.4 standard in terms of network throughput along with their life cycle. The results show that the $E-M A C_{I o T}$ provides balanced power consumption of the sensor nodes, increases data transmission up to $79 \%$ with $94 \%$ improved lifetime of the network as compared to the standard.

The organization of the paper is as:

Section 2 gives an overview of the IEEE 802.15.4 standard. Section 3 describes different MAC protocols for IoT both with and without energy harvesting techniques followed by the proposed work in 4 . The performance of the proposed scheme with the standard is evaluated in section 5, and section 6 concludes this 90 paper. 


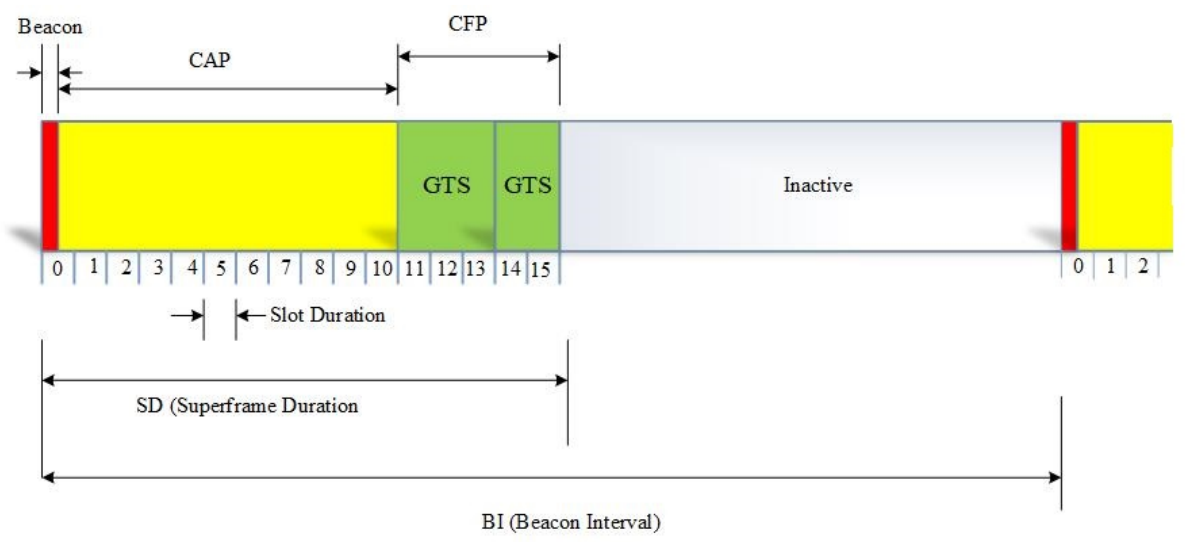

Figure 1: Superframe structure of IEEE 802.15.4 standard [19]

\section{An overview of IEEE 802.15.4 Standard}

The IEEE 802.15.4 standard is designed for Physical and MAC layers and operates in beacon and non-beacon enabled modes. During non-beacon enabled mode, nodes exchange their information in an ad-hoc manner. However, the beacon-enabled mode offers a superframe structure that comprises of an active and an inactive period. The active period starts with a beacon frame and followed by a Contention Access Period (CAP) and an optional Contention Free Period (CFP). PAN coordinator broadcasts the beacon frame by informing about CAP, CFP, and inactive period duration. During CAP, nodes send their all kind of requests by following the CSMA/CA method. During CFP, only selected nodes are allowed to explicitly send their data by allocating one or more Guaranteed Time Slots (GTS). The active period comprises of 16 equal duration slots, in which a maximum of 7 GTSs can be reserved during CFP. A complete superframe structure of IEEE 802.15.4 standard is shown in Fig. 1 .

Beacon enabled mode allows the necessities of energy utilization and QoS because it supports a flexible low duty cycle (DC). The duration from the start of one beacon to the start of the next beacon is known as Beacon Interval (BI) and the active period is known as Superframe Duration (SD). During the inactive period, all nodes keep their radios off to conserve energy. BI and SD 
are calculated from the following equations as:

$$
\begin{aligned}
& B I=960 \times 2^{B O} \text { symbols } \\
& S D=960 \times 2^{S O} \text { symbols }
\end{aligned}
$$

Each symbol duration of the standard in $2.4 \mathrm{GHz}$ frequency band is $16 \mu \mathrm{Sec}$. The duty cycle is given as:

$$
D C=S D / B I=2^{S O-B O}
$$

where $0 \leq S O \leq B O \leq 14[19]$.

Nodes preferred to send their data during CFP in their allocated GTS, as there is no collision. The standard allows the PAN coordinator to allocate GTS to only member nodes. A GTS requesting node first find out the number of GTS required $\left(G T S_{r e q}\right)$ in transmitting its data $L$, that can be calculated as with the help of the following equation:

$$
G T S_{r e q}=\left\lceil L / G T S_{t}\right\rceil
$$

here, $G T S_{t}$ represents the slot capacity. All nodes send their $G T S_{r e q}$ to the PAN coordinator during CAP. PAN coordinator after receiving all these requests allocates GTS on First Come First Serve (FCFS) basis and informs all successful nodes about their starting slot in the next beacon frame. The successful nodes are allowed to send their data in their assigned GTS without interference of other nodes.

\section{Related Work}

Limited energy in wireless sensor nodes is one of their major constraints. Nodes need to conserve their energy to increase their life cycle. Energy harvesting is used to increase the energy level of the nodes. Many researchers proposed 
different MAC protocols to improve the energy efficiency of IoT based WSNs. algorithm. In 27] probabilistic polling approached is used in which the sink varies the contention window to change the harvesting dynamic and the nodes having residual energy below a certain threshold will not take part in the con- 
tention process to conserve its energy thus improve the energy efficiency and lifetime of the network. In [28] AH-MAC is proposed and is based on LowEnergy Adaptive Clustering Hierarchy (LEACH). In AH-MAC only the end nodes such as cluster heads are responsible for energy harvesting while other nodes are not equipped with energy harvesting circuits and are only battery operated. Consequently, the lifetime of the network is improved by pursuing most of the activities by cluster heads.

In 29] IW-MAC is proposed in which the energy of the sensor nodes is mostly consumed by during actual data transmission while the control packets consume less energy thus more data packets and less control overhead packets are transmitted thus improves the energy of the IoT based sensor nodes. In [30], a scalable energy-efficient scheme for green IoT based heterogeneous wireless nodes is proposed that divides the area into different zones and uses the relay nodes for better use of transmission. The relay nodes are selected using the election process by considering parameters such as residual energy, distance, and centrality. In 31] a duty cycle adjustment algorithm for IoT enabled precision agriculture is proposed which improves the energy consumption and throughput of the network. The duty cycle is adjusted using the residual energy of the sensor nodes. In [32] a self-sustainable RF energy harvesting algorithm (SS-RF) for IoT based WSN is proposed in which RF energy is harvested from LTE eNodeB and the energy harvesting period is adapted in accordance to incoming traffic load and harvested energy using Kalman filters.

Although the above proposed methods improve the energy efficiency of the IoT based wireless sensor networks but there are some issues that need to be addressed. Table 1 shows the comparative table between different MAC protocols proposed in literature as [20, 21, 24, 30, 31, 32] does not guarantee distribution of traffic based on their energy levels thus no Load Balancing is applied also the concept of Energy-aware deep sleep in which the nodes with low residual energy will go to sleep and harvest energy is not applied in [20, [21, [23, 24], [27, [28, 30, 32. Similarly, the concept of low energy devices to not take part in contention and the information about the next beacon transmission is missing 
in various proposed methods.

Table 1: Comparative table between different MAC

\begin{tabular}{|c|c|c|c|c|}
\hline Protocol & Load Balancing & Energy-aware deep sleep & Contention Reduction & Wake-up time awareness \\
\hline RF-MAC & NO & NO & YES & NO \\
\hline RF-ASSP & NO & NO & YES & YES \\
\hline ODMAC & YES & NO & YES & YES \\
\hline REACH & NO & NO & YES & NO \\
\hline AH-MAC & YES & NO & NO & NO \\
\hline IW-MAC & YES & NO & YES & NO \\
\hline SSES & NO & NO & YES & NO \\
\hline H.Agrawal et al & NO & YES & YES & NO \\
\hline SS-RF & NO & NO & YES & YES \\
\hline$E-M A C_{I o T}$ & YES & YES & YES & YES \\
\hline
\end{tabular}

IEEE 802.15.4 standard is highly attracted to IoT based WSNs due to its extremely low duty cycle. In this work, the proposed $E-M A C_{I o T}$ modifies this standard to improve the life cycle of the sensor nodes by offering load balancing by allowing nodes to remain in deep sleep when they are left with less energy.

\section{Proposed Methodology}

\subsection{System Model}

Inspired from [32], we consider a scenario as shown in Fig. 3 where a singlehop IoT network is in the proximity of LTE eNodeB. The IoT network consists of a central coordinator or sinks $\mathrm{C}$ and sensor nodes $\mathrm{N}=\left[\begin{array}{lll}1 & 2 \ldots . \mathrm{N}\end{array}\right]$. The sensor nodes are assumed to have RF-harvesting capabilities. The sensor node consists of a low-power RF transceiver, low-power micro-controller, RF energy harvester, power management unit, and energy storage. The low-power RF transceiver is responsible for data transmission and reception in the IoT sensor node while the RF harvester is used to harvest energy from the LTE eNodeB. The reason for using two different antennas for data and energy is the different band of frequencies for LTE and IoT network.

In this paper, the Physical Broadcast Channel (PBCH) and Physical Downlink Control Channel (PDCCH) of the LTE are used for the energy harvesting 


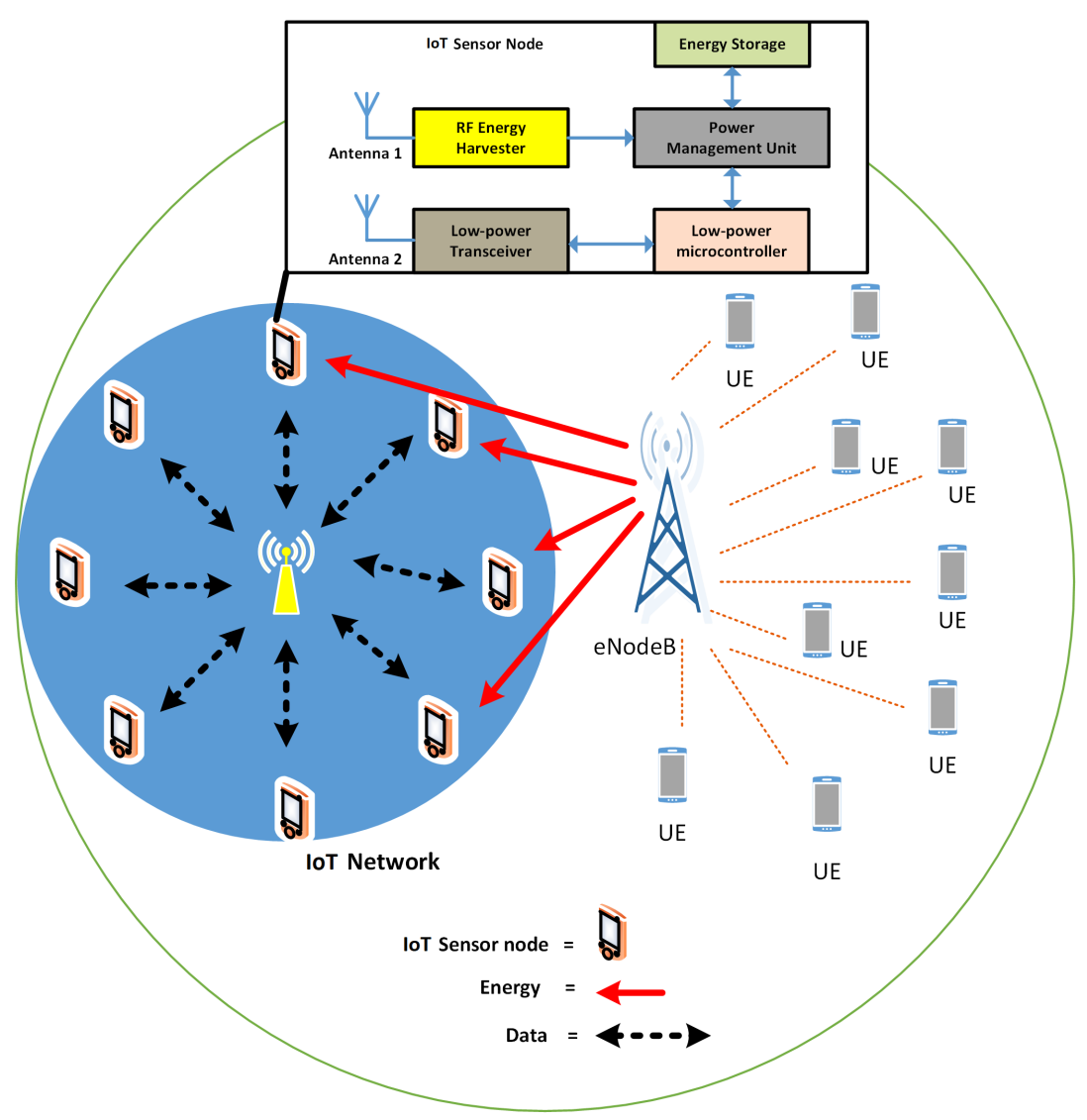

Figure 2: System Model which is divided into ten $1 \mathrm{~ms}$ sub-frames. The $1 \mathrm{~ms}$ sub-frame is further divided into two slots of $0.5 \mathrm{~ms}$ each. The $\mathrm{PBCH}$ is transmitted on the first four symbols of the first slot of the first sub-frame. The allocation of $\mathrm{PBCH}$ is spread over a duration of $40 \mathrm{~ms}$ (four radio frames) know as Transmission Time Interval 215 (TTI). Duration of one OFDM symbol is $\tau_{\text {sym }}=66.67 \mu$ s when normal prefix is used, so the $\mathrm{PBCH}$ is on 4 OFDM symbols and is spread over 4 radio frames resulting in a total length $\tau_{P B C H}$ of $16 \tau_{\text {sym }}$ in TTI.

The length of PDCCH depends on the number of OFDM symbols it has occupied. PDCCH is assigned to active UEs for controlling data transmis- 
220 depends on the number of active UEs requesting data transmission. Suppose $\gamma_{D}$ represents the level of traffic load on the LTE network. As an example if the traffic load on the LTE network is divided into three levels, $\gamma_{D}=\{1,2,3\}$. In a dense LTE scenario, the traffic load is high, so the value of $\gamma_{D}=3$. When the traffic load is average, $\gamma_{D}=2$ and for low traffic loads, $\gamma_{D}=1$. We assume that the traffic load in LTE remains the same for TTI $=40 \mathrm{~ms}$, hence $\gamma_{D}$ has a fixed value within a TTI. This yields the length of PDCCH $\tau_{P D C C H}=40 \gamma_{D} \tau_{\text {sym }}$ within a TTI 33$]$.

\subsection{RF Energy Harvesting}

230

odeB by the sensor nodes. Thus, we only consider the communication between the sensor nodes and the LTE eNodeB. In our model, the eNodeB is equipped with a single omnidirectional antenna and we also assume that the power control policy is not implemented on $\mathrm{PBCH}$ and $\mathrm{PDCCH}$. Let $P_{i}$ be the downlink transmit power of LTE eNodeB, $d$ be the distance between the sensor nodes and the eNodeB, and $a_{s i}$ be the channel coefficient that contains both the effects of large-scale path loss and small-scale Rayleigh fading. Thus, the unit amount of energy harvested at the sensor nodes as given by [21] will be equal to:

$$
e u=\zeta\left(\tau_{P D C C H}+\tau_{P B C H}\right) \frac{P_{i}\left|a_{s i}\right|^{2}}{d^{\alpha}}+\sigma^{2}
$$

where $\zeta$ is the efficiency of RF to DC and $\sigma^{2}$ denotes the noise power also the $\tau_{P B C H}$ and $\tau_{P D C C H}$ are the time duration of channels. As Rayleigh fading is assumed so the amplitude square of projection $a_{s i}$ is denoted by $\left|a_{s i}\right|^{2}$.

Now as the sensor node goes to sleep mode and will stop its transmission but will continue to harvest energy from LTE eNodeB harvested energy during the beacon interval will be equal to:

$$
E_{h}=\left(\frac{\tau_{\text {active }}+\tau_{\text {inactive }}}{\tau_{\text {frame }}}\right) \times e u
$$




$$
E_{h}=\left(\frac{\tau_{\text {active }}+\tau_{\text {inactive }}}{\tau_{\text {frame }}}\right) \times \zeta\left(\tau_{P D C C H}+\tau_{P B C H}\right) \frac{P_{i}\left|a_{s i}\right|^{2}}{d^{\alpha}}+\sigma^{2}
$$

where $\tau_{\text {active }}$ and $\tau_{\text {inactive }}$ are the active and inactive period of the sensor node and $\tau_{\text {frame }}$ is the TTI of LTE frame.

\subsection{Energy Consumption of Sensor Node}

A sensor node in a network consumes its energy during four modes such as, during data transmission $\left(E_{t x}\right)$, data receiving $\left(E_{r x}\right)$, during idle listening $\left(E_{\text {idle }}\right)$ and during sleep mode $\left(E_{\text {sleep }}\right)$. Total energy consumed by a sensor node is the sum of all these energies. $E_{t x}$ depends on the amount of transmitted data by nodes to other nodes as well as to the PAN coordinator. In the proposed work we does not include the energy model of the PAN coordinator and we assume that the PAN coordinator has sufficient energy source. In the reception mode, the sensor nodes receive data from the coordinator and the total energy consumed in this mode depends on the amount of data received by the sensor nodes. Nodes consume energy, when they are in idle listening mode by turning their transceivers in $O N$ position without transmitting or receiving any data. The more time, a node remains in idle listening mode, more energy it consumes and can not be ignored in energy calculation. The sleep mode is the one in which the sensor turns off its receiver and a very minute amount of energy is consumed. Due to its very small amount, it has been ignored in our calculation. The accumulated energy $\left(E_{A c c}\right)$ of a sensor node is calculated as:

$$
E_{\text {Acc }}=E_{t x}+E_{r x}+E_{\text {idle }}
$$

The energy consumed during the transmission mode can be given as

$$
E_{t x}=E_{G T S R E Q_{t x}}+E_{\text {Data }}{ }_{t x}
$$

where $E_{G T S R E Q_{t x}}$ is the energy consumed during a GTS request transmission and $E_{\text {Data }_{t x}}$ is the energy consumed while transmitting a data packet. $E_{G T S R E Q_{t x}}$ and $E_{D_{a t a} a_{t x}}$ can be further calculated as

$$
E_{G T S R E Q_{t x}}=V \times I_{t x} \times t_{t x}=V \times I_{t x} \times \frac{L_{G T S}}{R}
$$


where $V$ is the sensor battery voltage, $I_{t x}$ is the current consumed during the transmission, $L_{G T S}$ is the length of the GTS frame (equal to 9 bytes), $R$ is the data rate in bits per second (bps).

$$
E_{\text {Data }_{t x}}=V \times I_{t x} \times \frac{L}{R}
$$

here, $L$ is the data packet length in bits.

The energy consumption in the reception mode depends on the energy consumed during the beacon packet received by the sensor nodes. The length of the beacon packet depends on the number of nodes that have been allocated GTS and is given as:

$$
E_{r x}=V \times I_{r x} \times t_{r x}=V \times I_{r x} \times \frac{L_{\text {beacon }}}{R}
$$

where $I_{r x}$ is the current required during the reception and $t_{r x}$ is the receiving time that is the ratio of the length of beacon packet $L_{\text {beacon }}$ and data rate $R$.

The energy consumed during idle mode is given as

$$
E_{\text {idle }}=V \times I_{\text {idle }} \times t_{\text {idle }}
$$

where $I_{i d l e}$ is the current drawn during idle period. The duration of idle time $t_{\text {idle }}$ depends on the superframe duration (SD) and time consumed during transmission and reception of data and it is calculated as:

$$
t_{i d l e}=S D-t_{r x}-t_{t x}
$$

By exchanging values of equations $10,11,12$ and 13 in equation 8 the total energy consumed during a superframe is equal to:

$$
E_{\text {Acc }}=V\left[\left(\frac{L_{G T S}}{R}+\frac{L}{R}\right) I_{t x}+\left(t_{i d l e}\right) I_{i d l e}+\left(\frac{L_{\text {beacon }}}{R}\right) I_{r x}\right]
$$

\subsection{Residual Energy}

Wireless sensor nodes are powered by the battery. The residual energy of a node is its current battery capacity. The remaining life of a node depends upon 
its residual energy. If residual energy of a node $m$ before the start of $k \mathrm{BI}$ is $E_{k}^{m}$, then its residual energy during before the start of next BI $E_{k+1}^{m}$ is calculated as:

$$
E_{k+1}^{m}=E_{k}^{m}-E_{A c c-k}^{m}+E_{h-k}^{m}
$$

here, $E_{A c c-k}^{m}$ and $E_{h-k}^{m}$ are energies consumed and harvested by node $m$ in $k_{t h}$ BI. Energy of a WPAN before the start of $k$ BI $\left(E_{k}^{W P A N}\right)$ is the cumulative energy of all nodes in the WPAN. If WPAN comprises of $n$ nodes, then WPAN energy just before the start of $k+1 \mathrm{BI}\left(E_{k+1}^{W P A N}\right)$ is calculated as:

$$
E_{k+1}^{W P A N}=\sum_{i=1}^{i=k}\left(E_{k}^{i}-E_{A c c-k}^{i}+E_{h-k}^{i}\right)
$$

Based on these residual energies of WPAN nodes, $E-M A C_{I o T}$ proposes two algorithms as:

- One for the whole WPAN, that adjusts the duty cycle based on residual energy of WPAN.

- To check the residual energy of each data requesting node and preferring a higher residual energy node to send its data during CFP over lower energy level nodes.

\subsection{Duty Cycle Adjustment in $E-M A C_{I o T}$}

In this section, an algorithm is proposed that allows the PAN coordinator to adjust its duty cycle by evaluating the overall energy of the WPAN $\left(E_{P A N}\right)$ at the end of each $S D$. The algorithm allows the PAN coordinator to fine-tune the duty cycle of the next superframe without compromising the Quality of Service (QoS). The QoS is based on delay and number of collisions. The initial values of $\mathrm{SO}$ and $\mathrm{BO}$ are zeros.

If $E_{P A N}$ is less than the threshold energy level then algorithm reduces the duty cycle of the next BI to increase the sleeping time of nodes to conserve more energy in the following way:

- If QoS is satisfied then it increases the $B O$ without changing $S O$. 
- If QoS is compromised due to increased collisions, then it needs to increase $S O$ to increase the CAP duration with an increase in $B O$, so that the duty cycle should not increase.

- If QoS is compromised due to increased delay, then $S O$ is reduced without changing the $B O$.

If $E_{P A N}$ is in the permissible range, the algorithm only checks the QoS. In case QoS is satisfied then there will be no changes in the values of $S O$ and $B O$. However, if QoS is compromised then $S O$ and $B O$ will be adjusted as:

- If $\mathrm{QoS}$ is compromised only due to increased delay, then both $S O$ and $B O$ will be reduced to decrease the $B I$ time.

- If QoS is compromised only due to increased collisions, then $S O$ will be increased to increase the active period along with an increase in $B O$.

- If QoS is compromised due to both collisions and delay, then $S O$ is increased without changing $B O$.

These changes in parameter values of $S O$ and $B O$ are valid until they satisfy the standard's following limitations.

$$
\begin{gathered}
B O-S O \leq 10 \\
0 \leq S O \leq B O \leq 14
\end{gathered}
$$

A complete algorithm is shown in fig 3 .

\subsection{GTS Allocation Procedure in $E-M A C_{I o T}$}

In this section, the second algorithm of $E-M A C_{I o T}$ is proposed that allows the PAN coordinator to scrutinize GTS requesting nodes to send their data during GTS. This GTS allocation procedure in $E-M A C_{I o T}$ is based on individual energy level information of each requesting node and its amount of data traffic. 


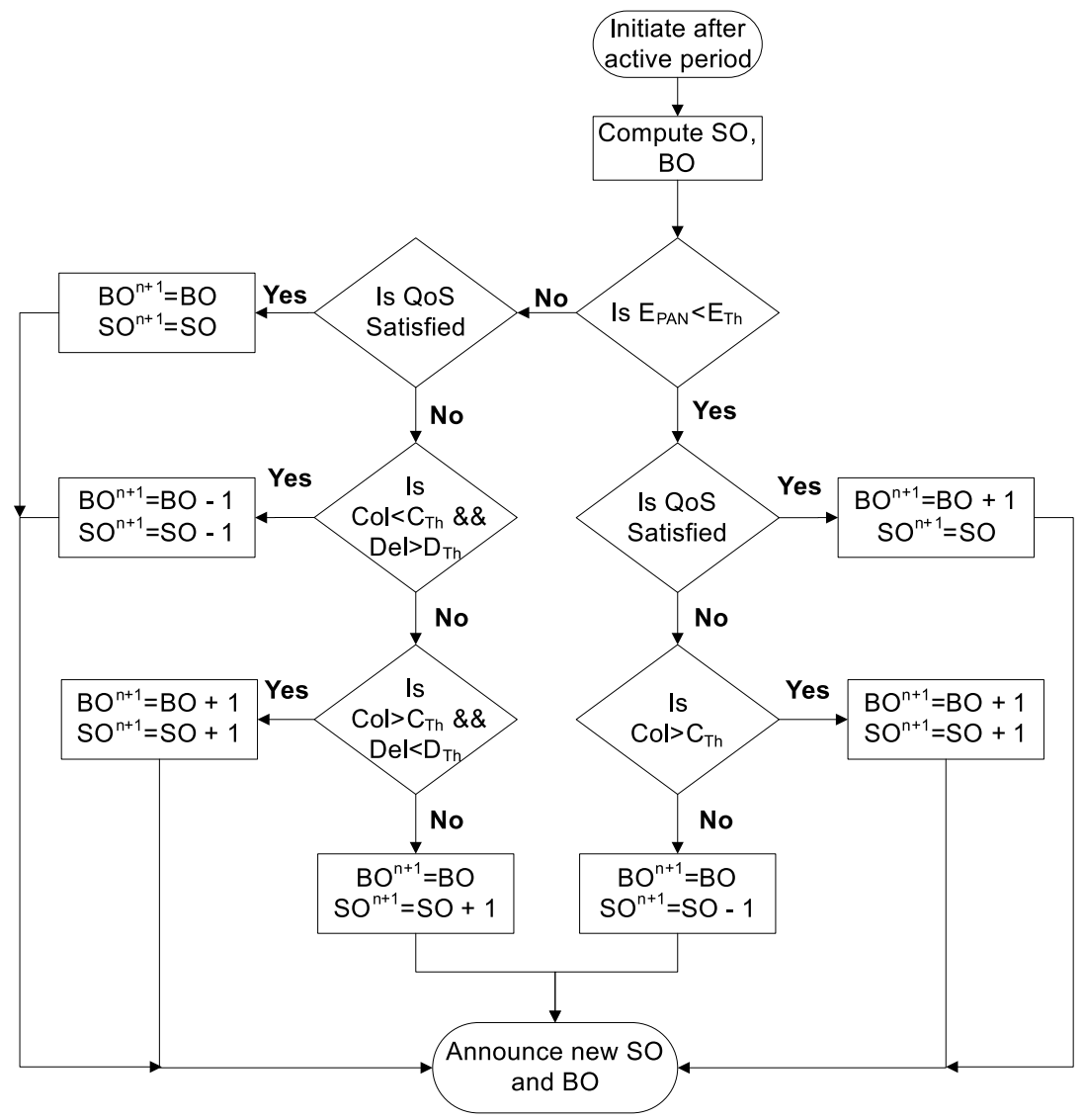

Figure 3: Adaptive duty cycle algorithm

\subsubsection{Energy Level Information}

In the proposed protocol, GTS requesting nodes to use the standard GTS request frame to request for GTS. The requesting nodes determine the number of GTS required to send information by knowing the data and the slot capacity $S_{c a p}$ given as

$$
G T S_{r e q}=D a t a / S_{c a p}
$$

In the GTS request frame, the starting 2 bytes define the control frame as shown in Fig. 4. In this figure, the bits $b_{7}, b_{8}$, and $b_{9}$ of the control frame of the GTS request frame are highlighted. These three bits were not used in the 
standard and were reserved for future work. That's why, in this work, these three bits are used to send nodes energy levels to the coordinator. These three bits allow nodes to divide the residual energy into 8 different levels as shown in Table 2. The coordinator allocates GTS to the requesting nodes by considering these energy levels.

Table 2: Residual Energy Information

\begin{tabular}{|c|c|}
\hline Energy Information Field Value & Energy Level \\
\hline 000 & Residual energy is $<12.5 \%$ \\
\hline 001 & Residual energy is $\geq 12.5 \% \&<25 \%$ \\
\hline 010 & Residual energy is $\geq 25 \% \&<37.5 \%$ \\
\hline 011 & Residual energy is $\geq 37.5 \% \&<50 \%$ \\
\hline 100 & Residual energy is $\geq 50 \% \&<62.5 \%$ \\
\hline 101 & Residual energy is $\geq 62.5 \% \&<75 \%$ \\
\hline 110 & Residual energy is $\geq 75 \% \&<87.5 \%$ \\
\hline 111 & Residual energy is $\geq 87.5 \% \&<100 \%$ \\
\hline
\end{tabular}

4.6.2. GTS Alloaction in $E-M A C_{I o T}$

In the IEEE 802.154 standards, the coordinator allocates GTS on First Come, First Serve (FCFS) basis without considering their energy levels. This could deplete the energy of some nodes, hence disconnecting them with the rest of the network. In the proposed protocol, the life cycle of the network is improved by scheduling prioritized transmissions to those GTS requesting nodes that have a higher energy level. This also allows nodes with lower energy consumption to harvest energy in the meantime and improve their battery residual energy.

To efficiently allocate GTS, the $E-M A C_{I o T}$ uses a knapsack optimization algorithm. Knapsack algorithm allows optimally collection of valuable items from the different available items up to its carrying capacity. The analogous mapping of this problem to select the GTS requesting nodes for slot allocation 


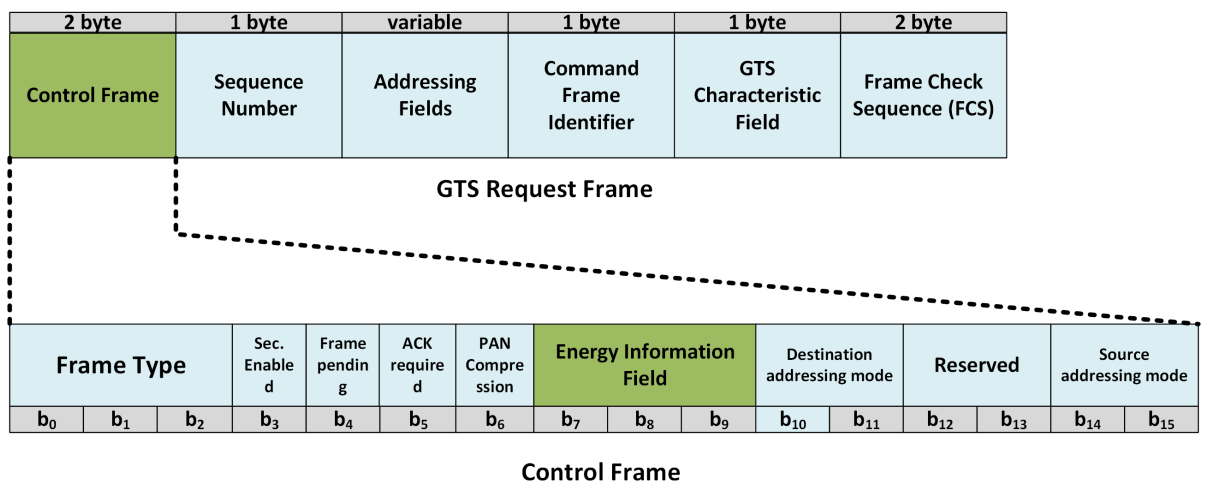

Figure 4: GTS Request Frame Format

Table 3: Mapping of GTS allocation problem to knapsack problem

\begin{tabular}{|c|c|c|}
\hline & GTS utilization with increased life cycle & Knapsack problem \\
\hline$M$ & Maximum number of available slots & Carrying capacity of knapsack \\
\hline$b$ & GTS requesting nodes to be satisfied GTS & Items to be packed \\
\hline$W_{b}$ & GTS requested by a node & weight of an item \\
\hline$P_{b}$ & Residual energy of a node & value of an item \\
\hline
\end{tabular}


is shown in Table 3. Here, the coordinator has to allocate the available GTS $M$ to the GTS requesting nodes $b$ in such a way that nodes with higher residual energy $P_{b}$ should be preferred for transmission. In this problem, the weights and the values of requesting nodes are the numbers of slots requested and the residual energy respectively. Optimal GTS allocation to the maximum number of nodes with higher energy levels to send their data can be mapped with the 0-1 Knapsack problem.

Knapsack allows us to select the most valuable items from the available list of items to fill its capacity. In our problem, knapsack allows us to optimally scrutinize those GTS requesting nodes which have better residual energies at the cost of more computation. Our problem for optimal selection of nodes by considering their residual energy levels are mapped with 0-1 knapsack problem as:

Let $W_{b}$ be the number of GTS requested by a node. If $W_{b} \leq \mathrm{M}$, then the sink allocates GTS to all GTS requesting nodes by applying the shortest job first algorithm [34. If $W_{b}>\mathrm{M}$, then the sink examines the nodes that request the GTS by applying the knapsack algorithm.

Table 4: Knapsack Table

\begin{tabular}{|c|c|c|c|c|c|c|c|c|}
\hline \multirow{2}{*}{ Sensor Nodes } & \multicolumn{7}{|c|}{ Maximum GTS Slots (7) } \\
\cline { 2 - 9 } & 0 & 1 & 2 & 3 & 4 & 5 & 6 & 7 \\
\cline { 2 - 9 } & 0 & 0 & 0 & 0 & 0 & 0 & 0 & 0 \\
\hline B & 0 & 8 & 8 & 8 & 8 & $\underline{\mathbf{8}}$ & 8 & 8 \\
\hline A & 0 & 8 & 8 & 16 & 16 & 16 & $\underline{\mathbf{1 6}}$ & 16 \\
\hline C & 0 & 8 & 8 & 16 & 16 & 16 & 16 & $\underline{\mathbf{2 1}}$ \\
\hline E & 0 & 8 & 8 & 16 & 16 & 16 & 16 & 21 \\
\hline D & 0 & 8 & 8 & 16 & 16 & 16 & 16 & 21 \\
\hline
\end{tabular}

Suppose there are 5 sensor nodes A, B, C, D, and E, which requested for 2, 1, 4, 5, and $4 \mathrm{CFP}$ slots along with their residual energy levels to the coordinator 
as $8,8,5,2$, and 4 respectively. It means there are 16 GTS requests against 7 GTS available in an SD.

As the number of requesting nodes is more than the available capacity, that is why, the coordinator has to scrutinize some nodes optimally by applying the knapsack optimization algorithm. The algorithm scrutinizes successful nodes by filling a knapsack table as follows:

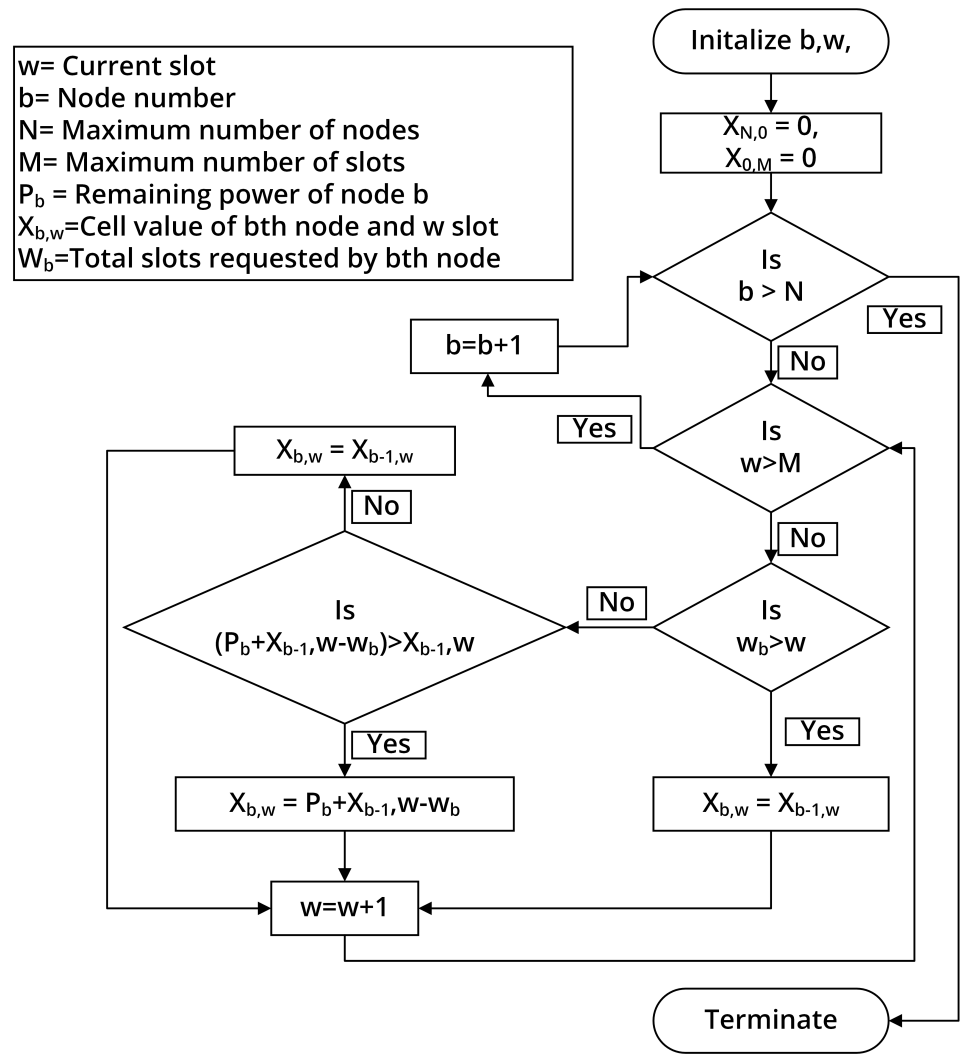

Figure 5: Knapsack table filling algorithm

1. Number of rows and columns of the knapsack table comprises the total number of GTS requesting nodes and GTS capacity respectively. The first row and first column of the table are initialized by all zeros.

2. Before filling the table, all the requesting nodes are placed in ascending order. That is, nodes with fewer GTS requesting slots fill their row ahead 
of other nodes as shown in Table 4 .

3. Each cell $(A[i, j])$ of the requesting node $A$ is filled with its immediate upper cell value $(A[i-1, j])$ till its requesting slots are more than current slot capacity. Otherwise, the cell will be filled with the value of $A[i-1, j]$ or sum of residual energy of that node and the value of a specific cell in the upper row.

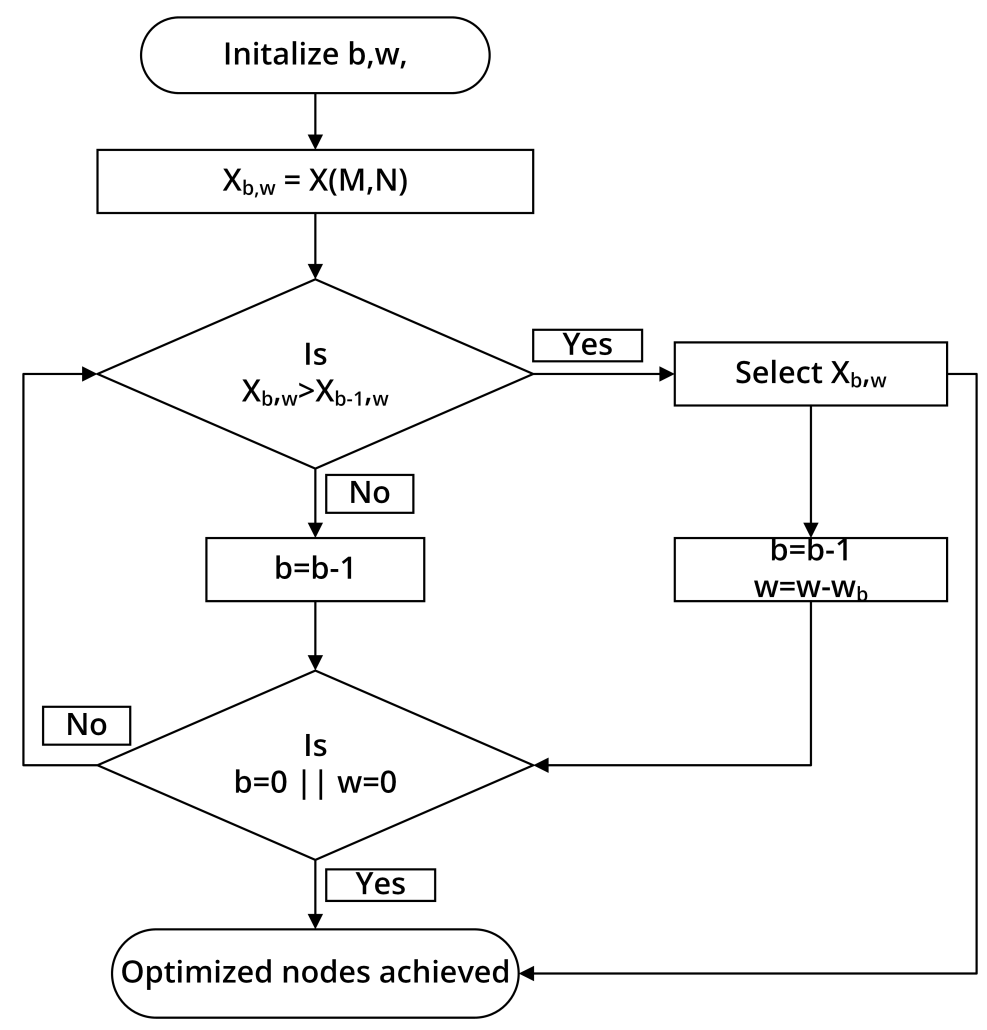

Figure 6: Optimal node selection algorithm

A complete algorithm to fill the knapsack table is shown in Fig. 5 The coordinator scrutinizes the nodes optimally by simply comparing the cell value with its upper cell value as described in the node selection algorithm shown in Fig. 6. A knapsack table for the given example is filled as shown in Table 4 Nodes A, B, and C are selected optimally in the given example due to their residual energy levels. 


\section{PERFORMANCE EVALUATION}

To evaluate the performance of $E-M A C_{I o T}$, a simulation environment is created as shown in Fig. 3 using MATLAB. We randomly deployed 15 sensor nodes within a radius of $100 \mathrm{~m}$ from the LTE eNodeB. The distance between the sensors nodes and the coordinator is kept $50 \mathrm{~m}$. For the propagation model, we use the two ray ground path loss model with a path loss exponent value of 2 and Rayleigh multipath fading. The transmit power of the LTE eNodeB is taken as $20 \mathrm{dBm}$, and the downlink channel frequency is $1.25 \mathrm{MHz}$. The simulation parameters are listed in Table. 5.

Table 5: Simulation Parameters

\begin{tabular}{|c|c|}
\hline Parameter & Value \\
\hline LTE eNodeB Tx Power & $20 \mathrm{dBm}$ \\
\hline Data Range (bytes) & $20-120$ \\
\hline Carrier frequency (IoT network) & $2400 \mathrm{MHz}$ \\
\hline Data rata (R) & $250 \mathrm{kbps}$ \\
\hline Battery & $3 \mathrm{~V}$ \\
\hline Sleeping current & $0.001 \mathrm{~mA}$ \\
\hline Receive current & $19.7 \mathrm{~mA}$ \\
\hline Transmit current & $17.4 \mathrm{~mA}$ \\
\hline LTE TTI & $40 \mathrm{~ms}$ \\
\hline LTE frame duration & $10 \mathrm{~ms}$ \\
\hline RF to DC efficiency & $0-0.7$ \\
\hline
\end{tabular}
by one i.e., the data generation request of the first sensor arrives first followed by the second sensor and so on. The number of GTS request by each sensor node is selected randomly. Based on the data requests and the shared energy level information by the sensor nodes, the coordinator runs the proposed protocol for 
802.15.4 MAC.

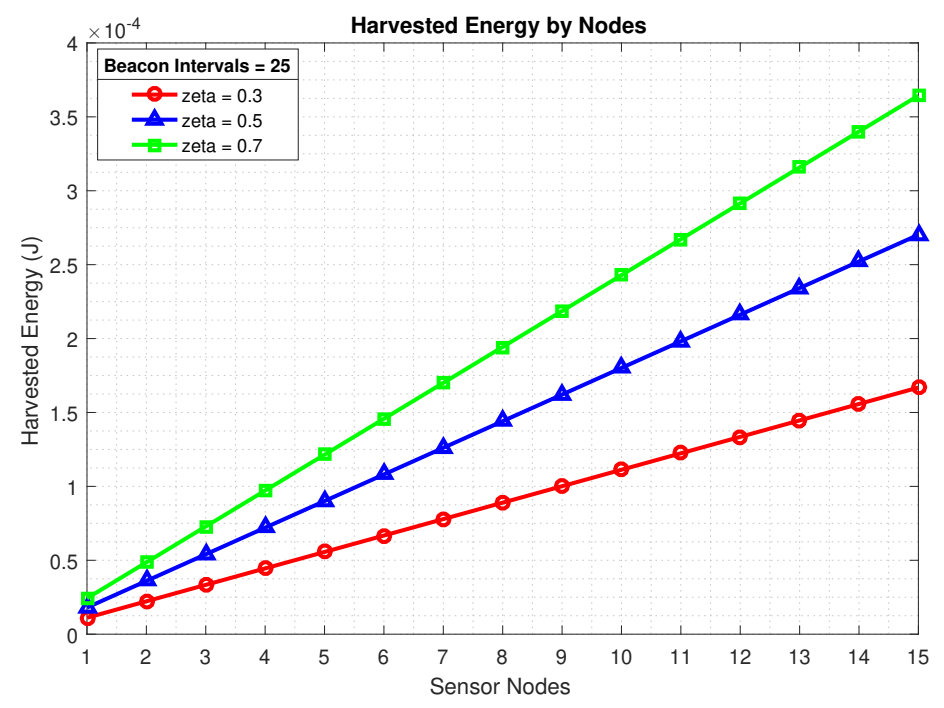

Figure 7: Effect of RF-to-DC rectification efficiency on the harvested energy

We first analyze the amount of energy harvested by the sensor nodes in the considered network scenario. In Fig. 7, the effect of RF-to-DC rectification efficiency $(\zeta)$ on harvested energy is shown for 25 beacon intervals considering three different values of $\zeta$ having same value of SO and BO. The SO and BO values are kept same to analyze the effect of $\zeta$ on harvested energy for proposed scheme. When the value of $\zeta$ is 0.3 , the accumulated harvested energy by sensor nodes is $165 \mu \mathrm{J}$. As we further increase $\zeta$ to 0.5 and 0.7 , the accumulated harvested energy is increased to 270 and $364 \mu \mathrm{J}$ respectively. Thus, the value of $(\zeta)$ directly impacts the amount of energy harvested during a certain period.

In Fig. 8 the impact of SO and BO values on harvested energy for 25 beacon intervals is shown. The SO and BO values determine the length of an active and inactive period of the total beacon interval. The duty cycle of a node is calculated as the ratio between the amount of time a node remains inactive period and the total time duration in a beacon interval. As in the proposed 


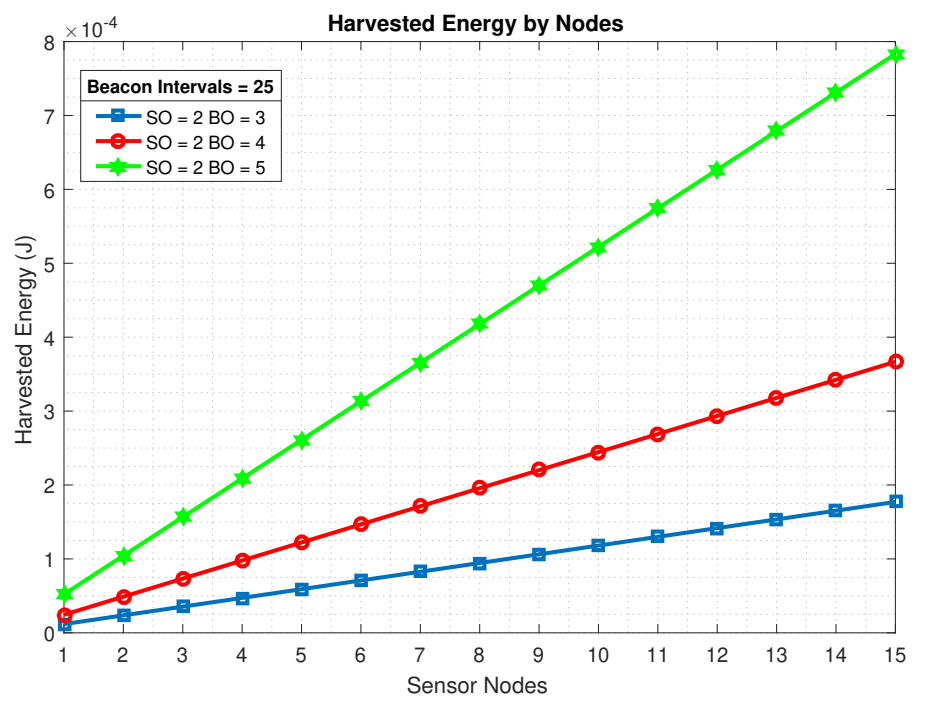

Figure 8: Effect of IoT sensor node's duty cycle on the harvested energy

scheme the sensor node harvest energy during the active and inactive period of the beacon interval so when the value of the $\mathrm{SO}$ and $\mathrm{BO}$ is 2 and 3 respectively corresponding to a $50 \%$ duty cycle, the amount of accumulated harvested energy is up to $177 \mu \mathrm{J}$. When we further increase the value of BO to 4 the inactive period is increased providing more time for the sensor to harvest energy thus the accumulated harvested energy is increased up to $366 \mu \mathrm{J}$ whereas, for the case of $\mathrm{SO}=2$ and $\mathrm{BO}=5$ with $12.5 \%$ duty cycle, the harvested energy is increased up to $783 \mu \mathrm{J}$.

Fig. 9 illustrate the average harvested energy at the IoT sensor nodes between standard and proposed for different values of SO and BO. It is evident from the graph that the proposed scheme outperforms the standard for different values of $\mathrm{SO}$ and $\mathrm{BO}$. When the value of $\mathrm{SO}$ and $\mathrm{BO}$ is 2 and 3 respectively the accumulated harvested energy for the standard is $127 \mu \mathrm{J}$ while in the case of the proposed scheme is $261 \mu \mathrm{J}$. As we further increase the value of SO and BO to 3 and 4 respectively the harvested energy for the standard is increased to $228 \mu J$ and for proposed the harvested energy is increased up to $364 \mu J$. The 


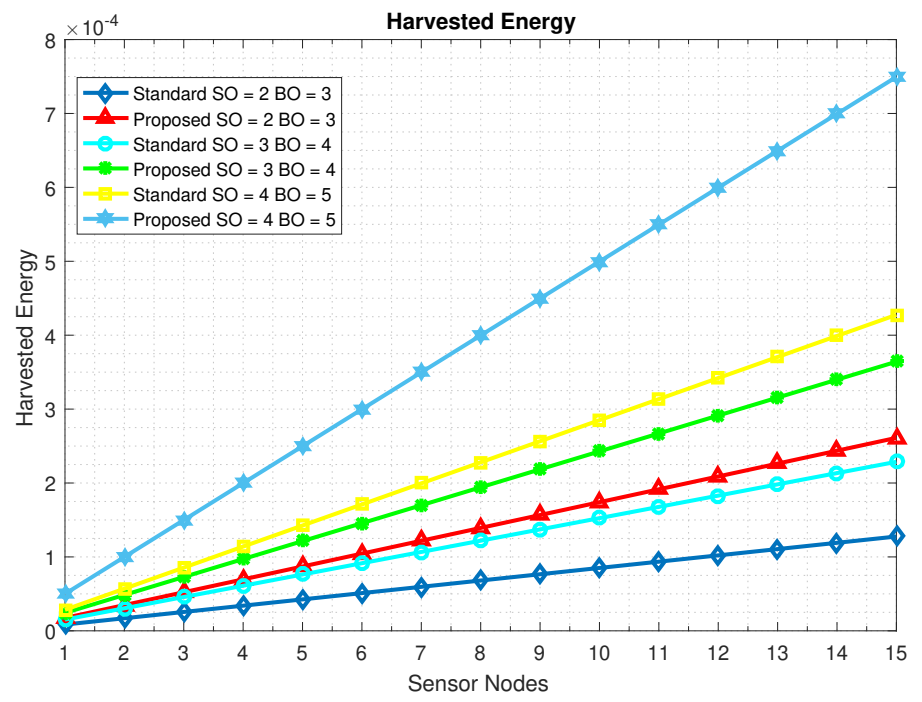

Figure 9: Average Harvested energy at IoT sensor nodes

standard uses the FCFS algorithm resulting in quick depletion of batteries thus the average harvested energy is low as compared to a proposed scheme that provides balanced energy consumption.

The results shown in Fig. 10 compares the performance of $E-M A C_{I o T}$ with the IEEE 802.15.4 standard in terms of energy consumption by the sensor nodes. As illustrated in the bar graph, the energy consumption in the standard for the first seven sensor nodes is higher than other nodes in the network. The reason is that the coordinator allocates GTS to the first four nodes more frequently because their data requests were received earlier. Based on the FCFS algorithm, the first four nodes get prioritized transmissions resulting in quick exhaustion of their batteries as compared to the other sensor nodes. On the other hand, in $E-M A C_{I o T}$ the coordinator selects the nodes having higher energy levels and avoids GTS allocation to the nodes having low energy levels. As a result, $E-M A C_{I o T}$ provides balanced energy consumption of all the sensor nodes, not causing any single sensor to deplete energy quickly.

Fig. 11 depicts the residual energy of all sensor nodes in the network for the 


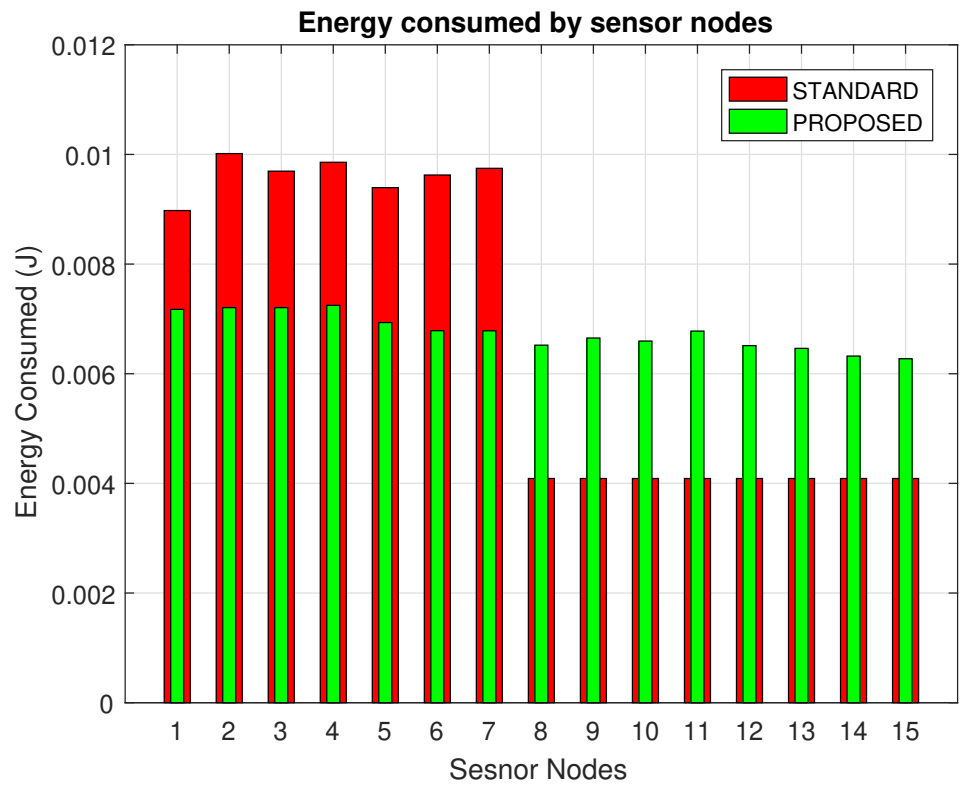

Figure 10: Energy consumption of the IoT sensor nodes

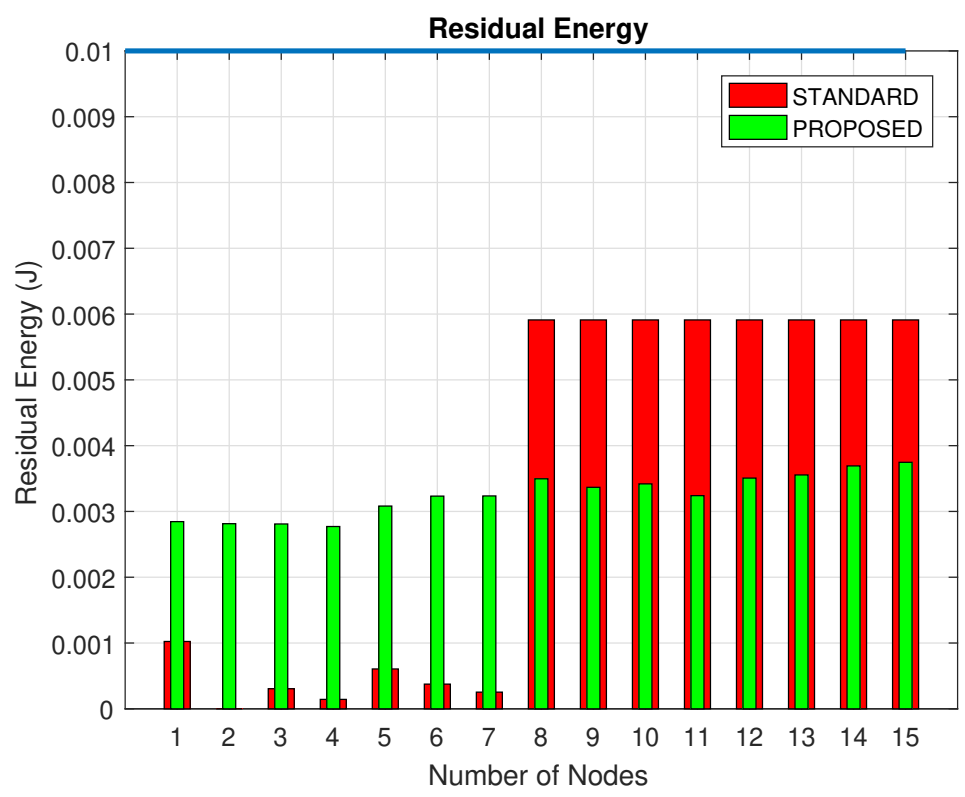

Figure 11: Residual energy of the IoT sensor nodes 
standard protocol and $E-M A C_{I o T}$. The initial energy of each sensor node was 0.1 joule. As the standard scheme allocates GTS on a FCFS basis, thus the residual energy of the first seven sensors are almost depleted. However, in $E-M A C_{I o T}$, residual energy of all nodes is available because knapsack prefers nodes with more residual energy to send their data over other nodes.

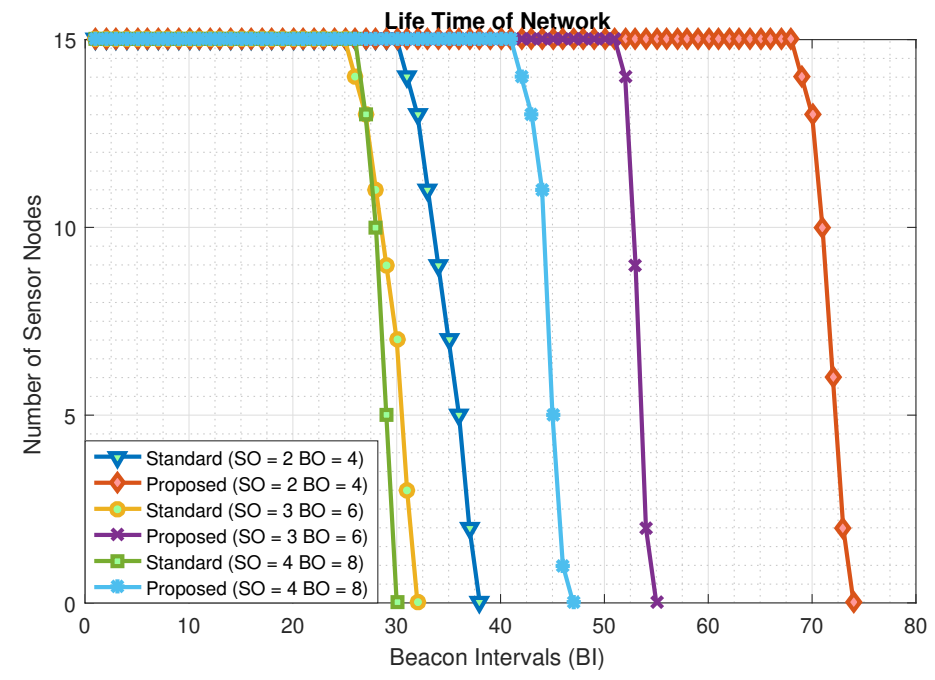

Figure 12: Life time of IoT sensor nodes

Fig. 12 shows the number of sensor nodes when their energy depletes at a particular $B I$. We consider the $B I$ value at which the battery of all the sensor nodes gets depleted as the battery lifetime of the IoT network. We show the results for the standard and $E-M A C_{I o T}$ for three different duty cycle values. For the standard protocol, the battery of all sensor nodes depletes within 38 $\mathrm{BI}$ for all three duty cycles. In comparison, $E-M A C_{I o T}$ extends the battery lifetime of the sensor nodes by $17-38$ BI. This highlights a key advantage of the proposed protocol which is to improve the battery lifetime of the IoT network.

Fig. 13 depicts the total data transmitted by the sensor nodes at different beacon intervals for three different values of $\mathrm{SO}$ and $\mathrm{BO}$. It can be seen that $E-M A C_{I o T}$ can transmit 17-19.5 kilobytes more data as compared to the standard protocol within $90 \mathrm{BI}$. This is because the battery depletion rate in 


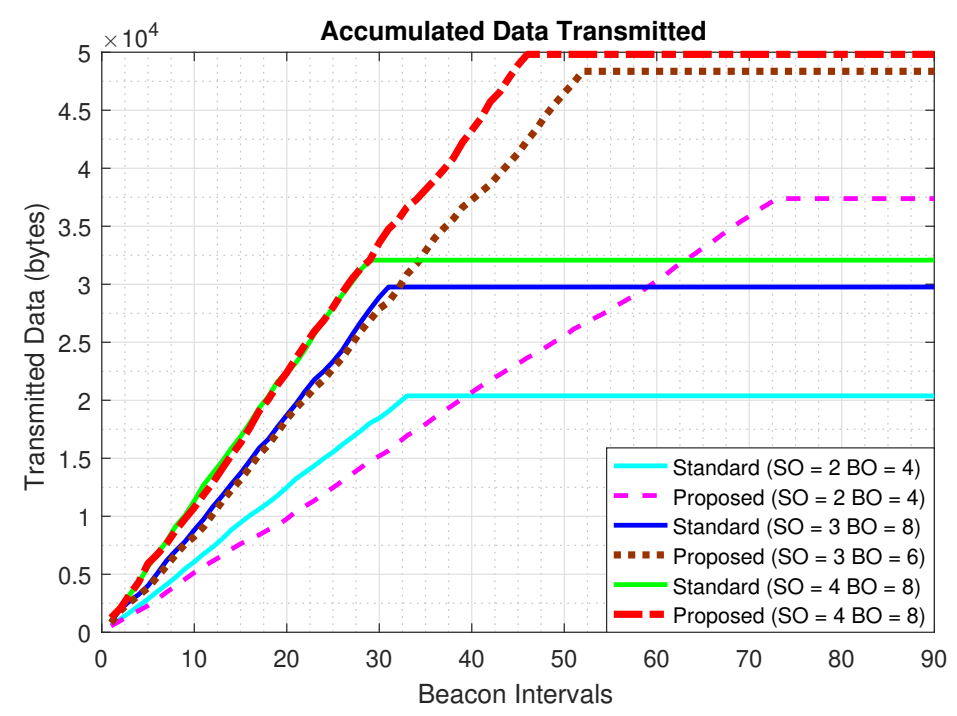

Figure 13: Data transmitted at different beacon intervals

the standard protocol is higher, thus resulting in a few sensor nodes to turn off.

On the other hand, $E-M A C_{I o T}$ provides balanced energy consumption and thus higher data throughput.

\section{Conclusion}

Energy-efficient GTS allocation with improved life cycle and better data transmission are major limitations in RF energy harvesting based IoT networks. IEEE 802.15.4 standard is widely used for medium access control in IoT networks. However, its data transmission during GTS results in unbalanced power consumption among nodes and less GTS utilization. The proposed protocol overcomes these limitations by adapting the duty cycle of IoT devices by considering their energy without compromising the QoS. Besides, it prefers higher residual energy nodes to send their data as compared to low energy nodes. The proposed protocol scrutinizes GTS requesting nodes optimally by modified knapsack algorithm. The algorithm adjusts nodes to allocate maximum available GTS and hence increases the throughput of the network. Those nodes, 


\section{References}

[1] Z. Zheng, T. Wang, J. Wen, S. Mumtaz, A. K. Bashir and S. H. Chauhdary, "Differentially Private High-Dimensional Data Publication in Internet of Things," in IEEE Internet of Things Journal, vol. 7, no. 4, pp. 2640-2650, April 2020

[2] G. Raja, A. Ganapathisubramaniyan, S. Anbalagan, S. B. M. Baskaran, K. Raja and A. K. Bashir, "Intelligent Reward-Based Data Offloading in Next-Generation Vehicular Networks," in IEEE Internet of Things Journal, vol. 7 , no. 5, pp. 3747-3758, May 2020

[3] Ali A, Khelil A, Shaikh F, Suri N. Efficient predictive monitoring of wireless sensor networks. Int J Auton Adapt Commun Syst 2012;5(3):233-54

[4] Ullah S, Higgins H, Braem B, Latre B, Blondia C, Moerman I, et al. A comprehensive survey of wireless body area networks. J Med Syst 2012;36 (3):1065-94

[5] W. Rafique, L. Qi, I. Yaqoob, M. Imran, R. u. Rasool and W. Dou, "Complementing IoT Services through Software Defined Networking and Edge Computing: A Comprehensive Survey," IEEE Communications Surveys \& Tutorials, Vol. 22 (3), pp. 1761-1804, Aug, 2020.

[6] J. M. Batalla, G. Mastorakis, C. X. Mavromoustakis and J. Zurek, "On 470 cohabitating networking technologies with common wireless access for home automation system purposes," in IEEE Wireless Communications, vol. 23, no. 5, pp. 76-83, October 2016. 
[7] Y. Liu, H. Dai, H. Wang, M. Imran, X. Wang, M. Shoaib, UAV-enabled Data Acquisition Scheme with Directional Wireless Energy Transfer for Internet of Things, Computer Communications, Vol. 155, pp. 184-196, Apr, 2020.

[8] Yalin Liu, Hong-Ning Dai, Qubeijian Wang, Mahendra K. Shukla, Muhammad Imran, Unmanned aerial vehicle for internet of everything: Opportunities and challenges, Computer Communications, vol. 155, pp. 66-83, 2020.

[9] Khan, S.; Alvi, A.N.; Javed, M.A.; Roh, B.-H.; Ali, J. An Efficient Superframe Structure with Optimal Bandwidth Utilization and Reduced Delay for Internet of Things Based Wireless Sensor Networks. Sensors 2020, 20, 1971.

[10] S. Ullah, M. Imran, M. A. Alnuem, "A Hybrid and Secure PriorityGuaranteed MAC protocol for Wireless Body Area Network", International Journal of Distributed Sensor Networks, Vol. 2014 (2014), Article ID 481761, Jan, 2014.

[11] Muddasir Rahim, Muhammad Awais Javed, Ahmad Naseem Alvi, Muhammad Imran,An efficient caching policy for content retrieval in autonomous connected vehicles, Transportation Research Part A: Policy and Practice,Volume 140,2020,Pages 142-152,

[12] F. Jameel, M. A. Javed and D. T. Ngo, "Performance Analysis of Cooperative V2V and V2I Communications Under Correlated Fading," in IEEE Transactions on Intelligent Transportation Systems, vol. 21, no. 8, pp. 34763484, Aug. 2020

[13] Muhammad Awais Javed, Sherali Zeadally, Zara Hamid, Trust-based security adaptation mechanism for Vehicular Sensor Networks, Computer Networks, Volume 137, 2018, Pages 27-36,

[14] M. A. Javed and J. Y. Khan, "Performance analysis of a time headway based rate control algorithm for VANET safety applications," 2013, 7th 
International Conference on Signal Processing and Communication Systems (ICSPCS), Carrara, VIC, 2013, pp. 1-6

[15] M. Abdel-Basset, R. Mohamed, M. Elhoseny, A. K. Bashir, A. Jolfaei and N. Kumar, "Energy-Aware Marine Predators Algorithm for Task Scheduling in IoT-based Fog Computing Applications," in IEEE Transactions on Industrial Informatics

[16] N. Kumar et al., "Achieving Energy Efficiency and Sustainability in Edge/Fog Deployment," in IEEE Communications Magazine, vol. 56, no. 5, pp. 20-21, May 2018, doi: 10.1109/MCOM.2018.8360845.

[17] G. Raja, S. Anbalagan, G. Vijayaraghavan, P. Dhanasekaran, Y. D. AlOtaibi and A. K. Bashir, "Energy-Efficient End-to-End Security for Software Defined Vehicular Networks," in IEEE Transactions on Industrial Informatics

[18] M. A. Hussain, P. khan and Kwak kyung Sup, "WSN research activities for military application," 2009 11th International Conference on Advanced Communication Technology, Phoenix Park, 2009, pp. 271-274.

[19] IEEE Draft Standard for Local and Metropolitan Area Networks Part 15.4: Low Rate Wireless Personal Area Networks (LR-WPANs) amendment to the MAC sub-layer, IEEE P802.15.4e/D6.0 (Revision of IEEE Std 802.15.4-2006) (2011) 1-200.

[20] M.Y. Naderi, P. Nintanavongsa, K.R. Chowdhury, RF-MAC: A medium access control protocol for re-chargeable sensor networks powered by wireless energy harvesting, IEEE Trans. Wireless Commun. 13 (7) (2014) 3926-3937, doi:10.1109/TWC.2014.2315211.

${ }_{525}$ [21] T. D. Nguyen, J. Y. Khan, and D. T. Ngo, "An adaptive MAC protocol for RF energy harvesting wireless sensor networks," in Proceedings of the 59th IEEE Global Communications Conference, GLOBECOM 2016, pp. 1-6, December 2016. 
[22] S. Guo, F. Wang, Y. Yang, and B. Xiao, Energy-efficient cooperative for simultaneous wireless information and power transfer in clustered wireless sensor networks, IEEE Trans. Commun., vol. 63, no. 11, pp.44054417, Nov 2015

[23] X. Fafoutis, N. Dragoni, ODMAC: An On-demand MAC protocol for energy harvesting - wireless sensor networks, in: ACM Symp. Performance Evaluation Wireless Ad Hoc, Sensor, Ubiquitous Netw., Miami, FL, USA, 2011, pp. 49-56.

[24] Kim, Teasung, et al. "REACH: An efficient MAC protocol for RF energy harvesting in wireless sensor network." Wireless Communications and Mobile Computing 2017 (2017).

[25] C. X. Mavromoustakis, G. Mastorakis and J. Mongay Batalla, "A Mobile Edge Computing Model Enabling Efficient Computation Offload-Aware Energy Conservation," in IEEE Access, vol. 7, pp. 102295-102303, 2019.

[26] S. Jiang, J. Cao, H. Wu and Y. Yang, "Fairness-based Packing of Industrial IoT Data in Permissioned Blockchains," in IEEE Transactions on Industrial Informatics, 2020.

[27] Alvi, A.N., Khan, S., Javed, M.A., Konstantin, K., Almagrabi, A.O., Bashir, A.K. and Nawaz, R., 2019. OGMAD: Optimal GTS-allocation mechanism for adaptive data requirements in IEEE 802.15. 4 based Internet of Things. IEEE Access, 7, pp.170629-170639.

[28] Al-Sulaifanie, S. Biswas, B.Khorsheed Al-Sulaifanie, AH-MAC: adaptive hierarchical MAC protocol for low-rate wireless sensor network applications, J. Sens. 2017 (2017) 1-15

[29] Kirubakaran, M.K. and Sankarram, N. J Ambient Intell Human Comput (2018). https://doi.org/10.1007/s12652-017-0662-6.

555 [30] Abdul-Qawy, A.S.H. and Srinivasulu, T. J Ambient Intell Human Comput (2019) 10: 1571. https://doi.org/10.1007/s12652-018-0758-7 
[31] Agrawal, H., Dhall, R., Iyer, K.S.S. et al. J Ambient Intell Human Comput (2019).https://doi.org/10.1007/s12652-019-013592

[32] T. D. Nguyen, J. Y. Khan and D. T. Ngo, "A Self-Sustainable RF Energy Harvesting Algorithm for WSN-Based IoT Applications," GLOBECOM 2017 - 2017 IEEE Global Communications Conference, Singapore, 2017, pp. 1-6.

[33] H. Zarrinkoub, Understanding LTE with MATLAB from mathemactical modelling to simulation and prototyping. John Wiley \& Sons, Ltd,2014

565 [34] A. N. Alvi, R. Mehmood, M. T. Ahmed, M. Abdullah and S. H. Bouk, "Optimized GTS Utilization for IEEE 802.15.4 Standard," 2018 International Conference on Selected Topics in Mobile and Wireless Networking (MoWNeT), Tangier, 2018, pp. 125-130. 